\title{
Silencing TAK1 alters gene expression signatures in bladder cancer cells
}

\author{
JIMIN CHEN, NAN ZHANG, JIAMING WEN and ZHEWEI ZHANG \\ Department of Urology, Second Affiliated Hospital, School of Medicine, Zhejiang University, \\ Hangzhou, Zhejiang 310009, P.R. China
}

Received May 12, 2015; Accepted September 22, 2016

DOI: $10.3892 /$ ol.2017.5819

\begin{abstract}
The aim of the present study was to identify the differentially expressed genes (DEGs) that are induced by the silencing of transforming growth factor- $\beta$-activated kinase 1 (TAK1) in bladder cancer cells and to analyze the potential biological effects. Dataset GSE52452 from mutant fibroblast growth factor receptor 3 (FGFR3) bladder cancer cells transfected with control siRNA or TAKl-specific siRNA was downloaded from Gene Expression Omnibus. The DEGs between the two groups were identified using Limma package following data pre-processing by Affy in Bioconductor. Enrichment analysis of DEGs was performed using the Database for Annotation, Visualization and Integrated Discovery, followed by functional annotation using TRANSFAC, TSGene and TAG databases. Integrated networks were constructed by Cytoscape and sub-networks were extracted employing BioNet, followed by enrichment analysis of DEGs in the sub-network. A total of 43 downregulated and 21 upregulated genes were obtained. The downregulated genes were enriched in five pathways, including NOD-like receptor signaling pathway and functions related to cellular response. The upregulated genes were associated with cellular developmental processes. Transcription factor EGRl and 9 tumor-associated genes were screened from the DEGs. Among the DEGs, 10 hub nodes may represent important roles in the complex metabolic network, including EGFR, CYP3A5, MAP3K7, GSTA1, PTHLH, ALDH1A1, KCND2, EGR1, ARRB1 and ITPR1. Additionally, EGFR was correlated with ERBB2, GRB2 and PIK3R1, and these were enriched in ErbB signaling pathway and various cancer-associated pathways. Silencing TAK1 may decrease cellular response to chemical stimulus via downregulating CYP3A5, MAP3K7, GSTA1, ALDHIA1, ARRB1 and ITPR1; increase cancer cell development via upregulating $E G F R$,
\end{abstract}

Correspondence to: Dr Zhewei Zhang, Department of Urology, Second Affiliated Hospital, School of Medicine, Zhejiang University, 88 Jiefang Road, Hangzhou, Zhejiang 310009, P.R. China E-mail: zheweizhangzwz@163.com

Key words: bladder cancer, TAK1-specific siRNA, cellular response, cell development, metastasis
EGRI and PTHLH; and regulate cancer metastasis through EGFR, ERBB2, GRB2 and PIK3RI.

\section{Introduction}

Bladder cancer may be classified into two stages: Non-muscle-invasive disease of low grade, and progressive muscle-invasive disease of high grade, which may further deteriorate into metastatic cancer (1). This type of cancer predominantly occurs as urothelial cell carcinoma and ranks as the fourth most common malignancy and the eighth most common cause of cancer-associated mortality in men (2). Overexpressed or mutant fibroblast growth factor receptor 3 (FGFR3) may contribute to the development of the transformed phenotype of urothelial carcinoma (3), and the targeted inhibition of FGFR3 may thus prevent the development of superficial bladder cancer (4). The transcription factor nuclear factor $\kappa \mathrm{B}(\mathrm{NF} \kappa \mathrm{B})$ is an important mediator of the angiogenesis and metastasis of bladder cancer (5). Activating mutations in FGFR3 can promote NFKB transcriptional activity through the important mediator transforming growth factor- $\beta$-activated kinase $1(T A K 1)$, which exerts a positive regulatory effect on the activity of $\mathrm{NF} \kappa \mathrm{B}$ in urothelial cell carcinoma (6). However, the exact regulatory effect of $T A K 1$ on the progression of bladder cancer remains unclear.

RNA interference may effectively aid in defining the roles of specific genes in the progression of cancer when specifically designed siRNAs are used to silence target genes $(7,8)$. Using the microarray expression data of bladder cancer cells transfected with control siRNA or TAKI-specific siRNA which were deposited by Salazar et al (6), the present study aimed to identify the differentially expressed genes (DEGs) and to screen for tumor-associated DEGs, followed by identification of the biological processes or pathways implicated by DEGs and the hub nodes in the complex protein-protein interaction (PPI) network and sub-network, in an attempt to provide a deeper insight into the molecular mechanisms mediating the effect of silencing $T A K 1$ on bladder cancer.

\section{Materials and methods}

Gene expression profiles. The gene expression profiles (accession number GSE52452) (6) from 6 samples of MGHU3 (Y375C) mutant FGFR3 bladder cancer cells that 
Table I. Significantly enriched KEGG pathways of upregulated and downregulated genes.

\begin{tabular}{lll}
\hline KEGG pathway & P-value & Gene list \\
\hline Downregulated genes & & \\
Aldosterone-regulated sodium reabsorption & 0.0079126 & SCNN1G, SGK1 \\
NOD-like receptor signaling pathway & 0.014731 & MAP3K7, TNFAIP3 \\
Retinol metabolism & 0.0177602 & ALDH1A1, CYP3A5 \\
Metabolism of xenobiotics by cytochrome P450 & 0.0215999 & CYP3A5, GSTA1 \\
Drug metabolism-cytochrome P450 & 0.0227555 & CYP3A5, GSTA1 \\
Upregulated genes & & \\
Cytokine-cytokine receptor interaction & 0.018547 & EGFR, IL18 \\
\hline
\end{tabular}

KEGG, Kyoto Encyclopedia of Genes and Genomes.

were transfected with control siRNA (GSM1267159-61) or TAK1-specific siRNA (GSM1267150-52), were downloaded from the public functional genomics data repository of Gene Expression Omnibus (9). These 6 samples (3 control samples vs. 3 TAK1 siRNA-treated samples) were previously researched using Affymetrix Human Gene 1.0 ST Array.

Data processing and screening of DEGs. The raw expression data were processed through RMA background correction (10), quantile normalization, $\log$ base $2\left(\log _{2}\right)$ transformation and probeset summarization to obtain the gene expression matrix by employing Affy package in Bioconductor (11) and probe annotation files provided by Brain Array Lab (brainarray.mbni. med.umich.edu/). Subsequently, the lists of DEGs between control and TAK1-specific siRNA-treated cells were generated under the thresholds of $\mid \log _{2}$ fold change (FC) $\mid \geq 1$ (fold-change magnitude, $>2$ ) and $\mathrm{P}$-value $<0.05$ using the empirical Bayes method offered by Limma package (12).

Functional enrichment analysis of DEGs. To provide insight into the biological functions or pathways involving the identified DEGs, the Gene Ontology (GO) Biological Process (13) and Kyoto Encyclopedia of Genes and Genomes (KEGG) (14) pathway enrichment analyses of DEGs were performed with the criterion of $\mathrm{P}<0.05$ using the Database for Annotation, Visualization and Integrated Discovery (15).

Functional annotations of DEGs. To identify the specific functions of DEGs, transcription factors were screened from the DEGs based on the TRANSFAC database (16), accompanied by screening of tumor-suppressor genes (TSGs) using the TSGene (17) database and identification of oncogenic genes using the tumor-associated genes (TAG) database (18).

Construction of PPI network and screening of sub-network. In consideration of the gene-gene interactions in the complex biological systems, the identified DEGs were inputted into the Search Tool for the Retrieval of Interacting Genes (STRING) database (19) to identify the interacting pairs with a combined score $>0.9$ (data downloaded on May 9, 2014). The PPI network was constructed using Cytoscape software (20), followed by excavation of the sub-network using the BioNet tool (bionet.bioapps.biozentrum.uni-wuerzburg.de/). KEGG pathway enrichment analysis of DEGs in the sub-network was performed to identify the associated biological pathways.

\section{Results}

Screened DEGs. With the criteria of $\log _{2} \mathrm{FCl} \geq 1$ and $\mathrm{P}<0.05$, a total of 43 downregulated genes and 21 upregulated genes were identified in the mutant FGFR3 bladder cancer cells transfected with $T A K 1$-specific siRNA in comparison with the cells transfected with control siRNA.

Functional enrichment results of DEGs. By performing a KEGG pathway enrichment analysis of the separate upregulated and downregulated genes, it was demonstrated that the downregulated genes were significantly enriched in five pathways, including the NOD-like receptor signaling pathway $[\mathrm{P}=0.014731$; mitogen-activated protein kinase kinase kinase 7 (MAP3K7) and tumor necrosis factor $\alpha$-induced protein 3 (TNFAIP3)] and the upregulated genes were enriched in the cytokine-cytokine receptor interaction pathway $[\mathrm{P}=0.018547$; epidermal growth factor receptor (EGFR) and interleukin 18 (IL18)] (Table I). The GO Biological Process enrichment analysis revealed that the downregulated genes were associated with cellular responses, including regulation of catalytic activity $\left[\mathrm{P}=5.45 \times 10^{-4}\right.$; e.g. aldehyde dehydrogenase 1 family member A1 (ALDH1A1), $\beta$-arrestin (ARRBl), inositol trisphosphate receptor (ITPRI) and $M A P 3 K 7)]$ and cellular response to chemical stimulus $\left[\mathrm{P}=1.38 \times 10^{-3}\right.$; e.g. ALDH1A1, ARRB1, ITPR1, MAP3K7, cytochrome P450 family 3 subfamily A polypeptide 5 (CYP3A5) and glutathione S-transferase $\alpha 1$ (GSTA1)]; the upregulated genes were associated with cellular developmental processes $\left[\mathrm{P}=5.80 \times 10^{-4}\right.$; e.g. $E G F R$, early growth response $1(E G R l)$ and parathyroid hormone-like hormone $(P T H L H)]$ (Table II).

Results of functional annotation of DEGs. Using TRANSFAC, TSGene and TAG databases, the functional annotation analysis screened 1 transcription factor (upregulated EGRl) and 9 TAGs, including 1 oncogene (upregulated EGFR), 5 TSGs (downregulated TNFAIP3, ras association domain family member 5 and carcinoembryonic antigen related cell adhesion molecule 7; upregulated GLI pathogenesis related 1 and $E G R I$ ) and 3 other TAGs (downregulated breast carcinoma 
Table II. Top 10 significantly enriched GO terms of upregulated and downregulated genes.

GO term P-value Gene list

Downregulated genes

GO:0006805 xenobiotic metabolic process

GO:0050790 regulation of catalytic activity

GO:0071466 cellular response to xenobiotic stimulus

GO:0009410 response to xenobiotic stimulus

GO:0045824 negative regulation of innate immune response

GO:0032715 negative regulation of interleukin-6 production

GO:0065009 regulation of molecular function

GO:0070887 cellular response to chemical stimulus

GO:0002819 regulation of adaptive immune response

GO:0018149 peptide cross-linking

Upregulated genes

GO:0036296 response to increased oxygen levels

GO:0055093 response to hyperoxia

GO:0030324 lung development

GO:0030323 respiratory tube development

GO:0060541 respiratory system development

GO:0001945 lymph vessel development

GO:0060571 morphogenesis of an epithelial fold

GO:0048869 cellular developmental process

GO:0009725 response to hormone stimulus

GO:0071320 cellular response to cAMP
5.32E-04 ACSL1, ALDH1A1, CYP3A5, GSTA1

5.45E-04 ACER2, ACSL1, ALDH1A1, ARRB1, DUSP10, FGD3, ITPR1, MAP3K7, SGK1, SYTL2, TNFAIP3

5.45E-04 ACSL1, ALDH1A1, CYP3A5, GSTA1

5.85E-04 ACSL1, ALDH1A1, CYP3A5, GSTA1

5.89E-04 DUSP10, TNFAIP3

9.53E-04 ARRB1, TNFAIP3

1.07E-03 ACER2, ACSL1, ALDH1A1, ARRB1, BAMBI, DUSP10, FGD3, ITPR1, MAP3K7, SGK1, SYTL2, TNFAIP3

1.38E-03 ACER2, ACSL1, ALDH1A1, ARRB1, BAMBI, CYP3A5, FGD3, GDF15, GSTA1, ITPR 1, MAP3K7, TNFAIP3

2.02E-03 DUSP10, MAP3K7, TNFAIP3

2.08E-03 SPRR1A, SPRR3

1.01E-06 EGR1, IL18, PDPN

1.01E-06 EGR1, IL18, PDPN

2.45E-05 HEG1, IL18, PDPN, PTHLH

2.64E-05 HEG1, IL18, PDPN, PTHLH

4.12E-05 HEG1, IL18, PDPN, PTHLH

2.81E-04 HEG1, PDPN

4.59E-04 EGFR, PTHLH

5.80E-04 ANTXR1, EGFR, EGR1, HEG1, IL18, MDK, MEA1, PDPN, PTHLH, STRADB

6.92E-04 EGFR, EGR1, IL18, MDK, STRADB

7.60E-04 EGR1, IL18

GO, Gene Ontology.

amplified sequence 1 and activin membrane-bound inhibitor; upregulated $P T H L H)$.

Analysis of the PPI network. Using the STRING database and Cytoscape tool, a PPI analysis of the DEGs was performed and an integrated PPI network was obtained. Based on this network, the top 10 hub nodes representing important roles in the complex metabolic networks were EGFR (degree, 178), CYP3A5 (degree, 83), MAP3K7 (degree, 58), GSTA1 (degree, 56), PTHLH (degree, 47), ALDH1A1 (degree, 45), potassium voltage-gated channel subfamily D member 2 (degree, 43), EGRl (degree, 42), ARRBl (degree, 36) and ITPRI (degree, 33) (Fig. 1). Additionally, this network revealed a correlation of MAP3K7 with TNFAIP3.

Analysis of the screened sub-network. Using the BioNet tool, a sub-network with $E G F R$ at the core was screened from the PPI network, in which EGFR was associated with v-erb-b2 avian erythroblastic leukemia viral oncogene homolog 2 (ERBB2), growth factor receptor-bound protein 2
(GRB2) and phosphoinositide-3-kinase, regulatory subunit 1 (PIK3R I) (Fig. 2). Furthermore, the DEGs in the sub-network were found to be enriched in various pathways associated with cancer, including the ErbB signaling pathway $\left(\mathrm{P}=3.07 \times 10^{-7}\right.$; e.g. EGFR, ERBB2, GRB2 and PIK3R1), pathways in cancer $\left(\mathrm{P}=5.90 \times 10^{-7}\right.$; e.g. EGFR, ERBB2, GRB2 and PIK3RI), prostate cancer $\left(\mathrm{P}=1.04 \times 10^{-4}\right.$; e.g. $E G F R, E R B B 2, G R B 2$ and $P I K 3 R 1)$, endometrial cancer $\left(\mathrm{P}=1.64 \times 10^{-4} ; E G F R, E R B B 2\right.$, $G R B 2$ and $P I K 3 R 1)$, non-small cell lung cancer $\left(\mathrm{P}=1.90 \times 10^{-4}\right.$; EGFR, ERBB2, GRB2 and $P I K 3 R 1)$ and glioma $\left(\mathrm{P}=3.90 \times 10^{-4}\right.$; e.g. EGFR, GRB2 and PIK3RI) (Table III).

\section{Discussion}

By re-analyzing the microarray data from specific mutant FGFR3 bladder cancer cells using bioinformatic methods, the present study identified 64 genes with significantly altered expression between TAK1-specific siRNA-treated cells and the control siRNA-treated cells, including 9 TAGs. The downregulated genes were related to the NOD-like receptor 


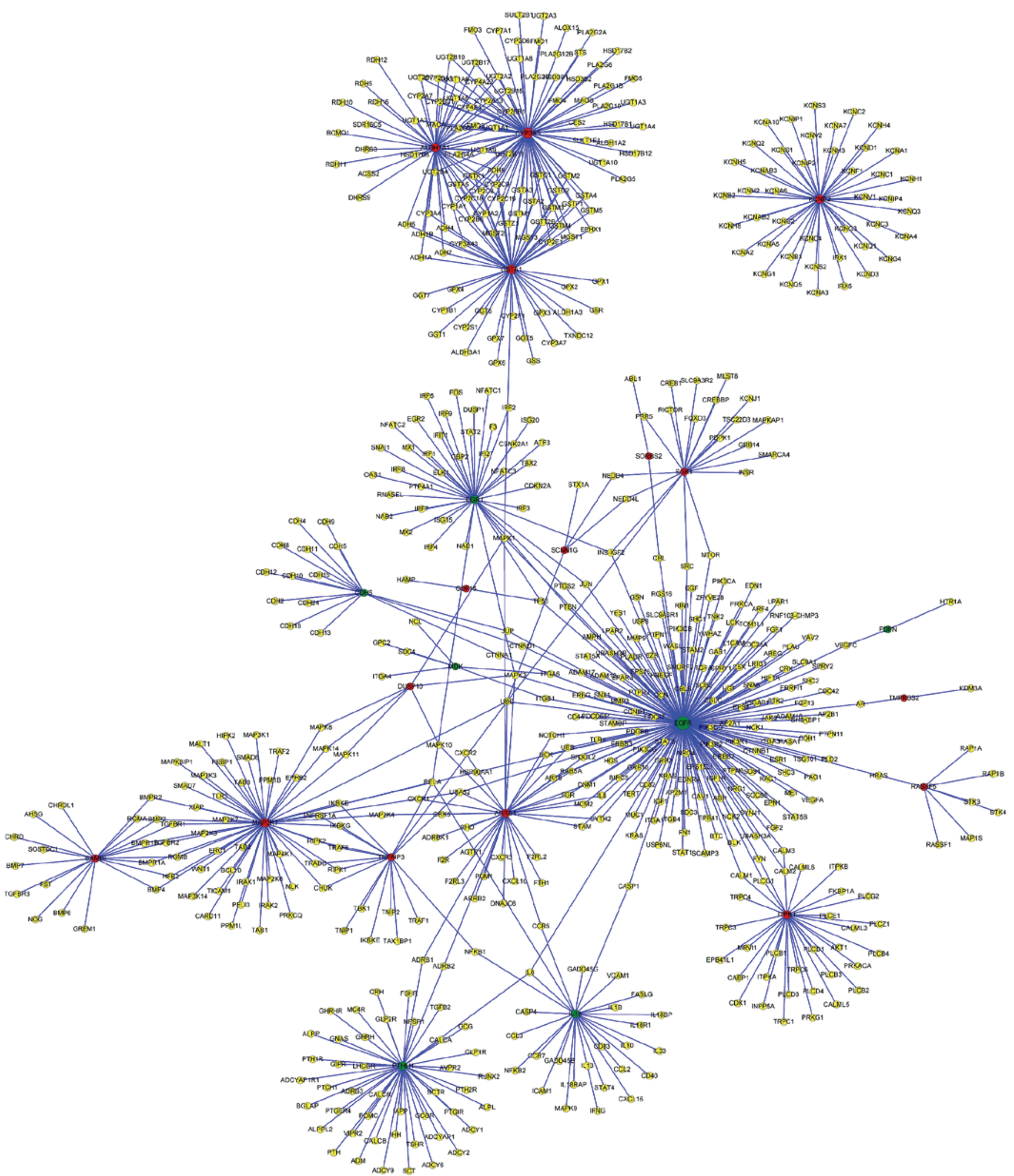

Figure 1. Protein-protein interaction network. Red represents upregulation, green represents downregulation, and yellow represents no difference.

signaling pathway and cellular response functions, whereas the upregulated genes were associated with cellular developmental processes. By constructing a PPI network, the present study identified 10 hub nodes that may exert the predominant effects on the network. Furthermore, the DEGs in the sub-network with $E G F R$ at the core were associated with various types of cancer and the ErbB signaling pathway.
TAK1 siRNA vs. control siRNA samples yielded 43 downregulated genes, of which 6 were identified to be hub nodes in the PPI network, including CYP3A5, MAP3K7, GSTA1, ALDHIA1, ARRB1 and ITPRI. CYP3A5 and GSTA1 are associated with the detoxification of chemical stimuli, electrophilic compounds or other damage stimuli $(21,22)$. They were also enriched in the term metabolism of xenobiotics by cytochrome 
Table III. Top 10 significantly enriched KEGG pathways of differentially expressed genes in the sub-network.

\begin{tabular}{|c|c|c|}
\hline KEGG pathway & P-value & Gene list \\
\hline ErbB signaling pathway & 3.07E-07 & $\begin{array}{l}\text { EGFR, ERBB2, GRB2, NRG1, HBEGF, ERBB3, } \\
\text { PIK3R1, EGFR, ITGA6, PTGS2, JUP, IGF1R, }\end{array}$ \\
\hline Pathways in cancer & $5.90 \mathrm{E}-07$ & $\begin{array}{l}\text { MET, FOS, } \\
\text { IL8, ERBB2, GRB2, PIK3R1 }\end{array}$ \\
\hline Focal adhesion & 7.34E-07 & $\begin{array}{l}\text { EGFR, ITGA6, CAV1, IGF1R, MET, VAV2, } \\
\text { ERBB2, GRB2, PIK3R1 }\end{array}$ \\
\hline Hepatitis C & $5.77 \mathrm{E}-06$ & $\begin{array}{l}\text { EGFR, STAT2, IRF1, IL8, GRB2, OAS1, } \\
\text { PIK3R1 }\end{array}$ \\
\hline Endocytosis & 8.07E-05 & $\begin{array}{l}\text { EGFR, ARRB1, AP2M1, CAV1, IGF1R, } \\
\text { MET, ERBB3 }\end{array}$ \\
\hline Prostate cancer & $1.04 \mathrm{E}-04$ & EGFR, IGF1R, ERBB2, GRB2, PIK3R1, \\
\hline Malaria & $1.52 \mathrm{E}-04$ & MET, IL8, SDC3, SDC4 \\
\hline Endometrial cancer & $1.64 \mathrm{E}-04$ & EGFR, ERBB2, GRB2, PIK3R1 \\
\hline Non-small cell lung cancer & $1.90 \mathrm{E}-04$ & EGFR, ERBB2, GRB2, PIK3R1 \\
\hline Glioma & $3.90 \mathrm{E}-04$ & EGFR, IGF1R, GRB2, PIK3R1 \\
\hline
\end{tabular}

KEGG, kyoto encyclopedia of genes and genomes.

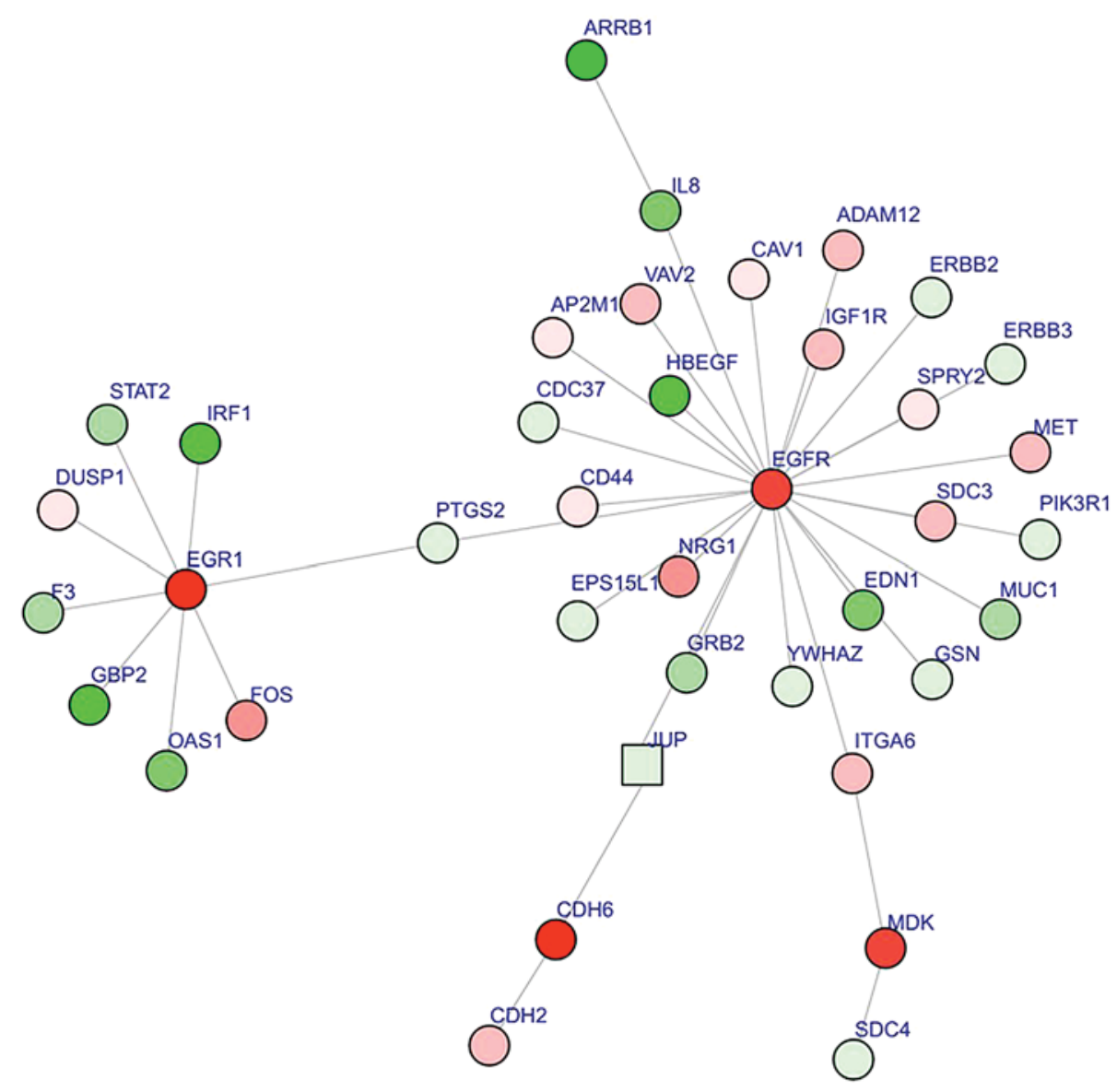

Figure 2. Extracted sub-networks. Color depth represents fold-change magnitude of differential expression. Red represents upregulation and green represents downregulation. Squares represent genes with low importance in the sub-network. Circles represent genes with high importance in the sub-network.

P450, which plays important roles in detoxification (23). $M A P 3 K 7$, which correlated with TNFAIP3, was enriched in the NOD-like receptor signaling pathway which is involved in sensing intracellular microbial motifs or other damage stimuli (24). ALDH1A1 and CYP3A5 were enriched in retinol metabolism; the loss of retinol acyltransferase is inversely 
correlated with the invasion bladder cancer (25). ARRBI is considered to cause a specific dampening cellular response to stimuli or sensory signals (26) and ITPRI mediates calcium release which then amplifies apoptosis in response to specific stimuli $(27,28)$. Accordingly, the present study further demonstrated that the 6 hub nodes in the PPI network were enriched in the GO function cellular response to chemical stimulus. It is therefore possible that silencing of TAKl using specific siRNA transfection may alter the cellular response-associated pathways or functions in response to stimuli via regulating the expression of the 6 DEGs.

TAK1 siRNA treatment induced 21 upregulated genes in bladder cancer cells, among which EGFR, PTHLH and $E G R 1$ were identified as hub nodes in the PPI network. These 3 genes were all enriched in cellular developmental processes. As exemplified, activated EGFR, which contributes to phenotypic characteristics in various tumor types (29), is an effective therapeutic target for the treatment of bladder cancer $(30,31)$. Increased expression of PTHLH resulting from downregulated p38MAPK signaling is associated with metastatic lesions to the liver and lung from colon cancer cells (32). EGR1 also exerts pro-tumorigenic effects by contributing to tumor infiltration, node formation and metastasis (33). Thus, the upregulation of these genes in TAK1 siRNA-treated bladder cancer cells may suggest that silencing TAKl has the effect of promoting cancer cell developmental processes. Furthermore, the present study also predicted EGFR, EGRI and PTHLH as TAGs, which suggests their potential use as therapeutic targets for the diagnosis and treatment of bladder cancer.

Using BioNet software, a sub-network with EGFR at the core was screened from the PPI network. According to the PPI network, $E G F R$ was correlated with $E R B B 2$, $G R B 2$ and PIK3R1. These 4 DEGs were enriched in various cancer types, including prostate cancer, endometrial cancer, non-small cell lung cancer and glioma. As previously reported, the $E G F R$ family of four receptors including $E G F R$ and $E R B B 2$, is implicated in the development and progression of various human cancer types (34). The activation of $E R B B 2$ may result in resistance to cetuximab-based therapy targeting $E G F R$, while the inhibition of this gene can restore cetuximab sensitivity in patients with cetuximab-resistant cancers (35). GRB2 amplification is observed in esophageal squamous cell carcinoma and is significantly involved in lymph node metastases (36). Phosphatidylinositol 3-kinase $(P I 3 K)$, which may promote cancer cell survival, is an important therapeutic target in cancer (37). PIK3RI, the inhibitory subunit of $P I 3 K$, often undergoes mutations in endometrial cancer $(38,39)$. The enrichment results also revealed the these 4 DEGs were enriched in ErbB signaling pathway, which is involved in regulating cell survival and adhesion (40). Silencing $T A K 1$ in bladder cancer cells led to the upregulation of EGFR and downregulation of ERBB2, GRB2 and $P I K 3 R 1$, suggesting an inhibitory effect of silencing TAK1 on the metastasis of bladder cancer cells via modulation of the ErbB signaling pathway. However, the explicit molecular mechanisms require further research.

In summary, the present study demonstrated that silencing TAK1 induced 43 downregulated and 21 upregulated genes. Silencing TAK1 may lead to decreased cellular response to chemical stimuli via downregulating $C Y P 3 A 5, M A P 3 K 7$, GSTAI, ALDHIAI, ARRBI and ITPRI, as well as increased cancer cell developmental processes via upregulating $E G F R$, EGRI and PTHLH. In addition, silencing TAK1 may exert regulatory effects on bladder cancer metastasis and other various cancer types via regulating the expression of $E G F R$, ERBB2, GRB2 and PIK3R1.

\section{Acknowledgements}

The present study was supported by the Zhejiang Provincial Natural Science Foundation (grant no., LY13H050001).

\section{References}

1. McCarthy N: Bladder cancer: Seemingly similar. Nat Rev Cancer 14: 214-215, 2014.

2. Siegel R, Ma J, Zou Z and Jemal A: Cancer statistics, 2014. CA Cancer J Clin 64: 9-29, 2014.

3. Tomlinson D, Baldo O, Harnden P and Knowles M: FGFR3 protein expression and its relationship to mutation status and prognostic variables in bladder cancer. J Pathol 213: 91-98, 2007.

4. Tomlinson DC, Hurst CD and Knowles MA: Knockdown by shRNA identifies S249C mutant FGFR3 as a potential therapeutic target in bladder cancer. Oncogene 26: 5889-5899, 2007.

5. Karashima T, Sweeney P, Kamat A, Huang S, Kim SJ, Bar-Eli M, McConkey DJ and Dinney CP: Nuclear factor-kappaB mediates angiogenesis and metastasis of human bladder cancer through the regulation of interleukin-8. Clin Cancer Res 9: 2786-2797, 2003.

6. Salazar L, Kashiwada T, Krejci P, Meyer AN, Casale M, Hallowell M, Wilcox WR, Donoghue DJ and Thompson LM: Fibroblast growth factor receptor 3 interacts with and activates TGF $\beta$-activated kinase 1 tyrosine phosphorylation and NFKB signaling in multiple myeloma and bladder cancer. Plos One 9: e86470, 2014.

7. Reynolds A, Leake D, Boese Q, Scaringe S, Marshall WS and Khvorova A: Rational siRNA design for RNA interference. Nat Biotechnol 22: 326-330, 2004.

8. Folini $M$, Pennati $M$ and Zaffaroni N: RNA interference-mediated validation of genes involved in telomere maintenance and evasion of apoptosis as cancer therapeutic targets. Method Mol Biol 487: 303-330, 2009.

9. Barrett T and Edgar R: Gene expression omnibus: Microarray data storage, submission, retrieval and analysis. Methods Enzymol 411: 352-369, 2006.

10. Irizarry RA, Hobbs B, Collin F, Beazer-Barclay YD, Antonellis KJ, Scherf U and Speed TP: Exploration, normalization and summaries of high density oligonucleotide array probe level data. Biostatistics 4: 249-264, 2003.

11. Gautier L, Cope L, Bolstad BM and Irizarry RA: Affy-analysis of affymetrix genechip data at the probe level. Bioinformatics 20: 307-315, 2004.

12. Smyth GK: Linear models and empirical bayes methods for assessing differential expression in microarray experiments. Stat Appl Genet Mol Biol 3: Article3, 2004.

13. Cheng L, Lin H, Hu Y, Wang J and Yang Z: Gene function prediction based on the gene ontology hierarchical structure. Plos One 9: e107187, 2014.

14. Altermann E and Klaenhammer TR: PathwayVoyager: Pathway mapping using the Kyoto encyclopedia of genes and genomes (KEGG) database. BMC Genomics 6: 60, 2005.

15. Dennis G JR, Sherman BT, Hosack DA, Yang J, Gao W, Lane HC and Lempicki RA: DAVID: Database for annotation, visualization and integrated discovery. Genome Biol 4: P3, 2003

16. Fogel GB, Weekes DG, Varga G, Dow ER, Craven AM, Harlow HB, Su EW, Onyia JE and Su C: A statistical analysis of the TRANSFAC database. Biosystems 81: 137-154, 2005.

17. Zhao M, Sun J and Zhao Z: TSGene: A web resource for tumor suppressor genes. Nucleic Acids Res 41: D970-D976, 2013.

18. Chen JS, Hung WS, Chan HH, Tsai SJ and Sun HS: In silico identification of oncogenic potential of fyn-related kinase in hepatocellular carcinoma. Bioinformatics 29: 420-427, 2013. 
19. Franceschini A, Szklarczyk D, Frankild S, Kuhn M, Simonovic M Roth A, Lin J, Minguez P, Bork P, von Mering C and Jensen LJ: STRING v9.1: Protein-protein interaction networks, with increased coverage and integration. Nucleic Acids Res 41: D808-D815, 2013.

20. Kohl M, Wiese S and Warscheid B: Cytoscape: Software for visualization and analysis of biological networks. Methods Mol Biol 696: 291-303, 2011

21. Lah L, Podobnik B, Novak M, Korošec B, Berne S, Vogelsang M, Kraševec N, Zupanec N, Stojan J, Bohlmann J and Komel R: The versatility of the fungal cytochrome P450 monooxygenase system is instrumental in xenobiotic detoxification. Mol Microbiol 81: 1374-1389, 2011.

22. Karpusas M, Axarli I, Chiniadis L, Papakyriakou A, Bethanis K, Scopelitou K, Clonis YD and Labrou NE: The interaction of the chemotherapeutic drug chlorambucil with human glutathione transferase A1-1: Kinetic and structural analysis. Plos One 8: e56337, 2013

23. Nebert DW and Dalton TP: The role of cytochrome P450 enzymes in endogenous signalling pathways and environmental carcinogenesis. Nat Rev Cancer 6: 947-960, 2006.

24. Fritz JH, Ferrero RL, Philpott DJ and Girardin SE: Nod-like proteins in immunity, inflammation and disease. Nat Immunol 7: 1250-1257, 2006.

25. Boorjian S, Tickoo SK, Mongan NP, Yu H, Bok D, Rando RR, Nanus DM, Scherr DS and Gudas LJ: Reduced lecithin retinol acyltransferase expression correlates with increased pathologic tumor stage in bladder cancer. Clin Cancer Res 10 3429-3437, 2004.

26. Wincent J, Schoumans J and Anderlid BM: De novo deletion of chromosome 11q13.4-q14.3 in a boy with microcephaly, ptosis and developmental delay. Eur J Med Genet 53: 50-53, 2010.

27. Ferris CD, Huganir RL, Supattapone S and Snyder SH: Purified inositol 1,4,5-trisphosphate receptor mediates calcium flux in reconstituted lipid vesicles. Nature 342: 87-89, 1989.

28. Boehning D, Patterson RL, Sedaghat L, Glebova NO, Kurosaki T and Snyder SH: Cytochrome c binds to inositol $(1,4,5)$ trisphosphate receptors, amplifying calcium-dependent apoptosis. Nat Cell Biol 5: 1051-1061, 2003.

29. Nicholson R, Gee J and Harper M: EGFR and cancer prognosis. Eur J Cancer 37 (Supply 4): S9-S15, 2001.
30. Black PC, Brown GA, Dinney CP, Kassouf W, Inamoto T, Arora A, Gallagher D, Munsell MF, Bar-Eli M, McConkey DJ and Adam L: Receptor heterodimerization: A new mechanism for platelet-derived growth factor induced resistance to anti-epidermal growth factor receptor therapy for bladder cancer. J Urol 185: 693-700, 2011.

31. Chadalapaka G, Jutooru I, Burghardt R and Safe S: Drugs that target specificity proteins downregulate epidermal growth factor receptor in bladder cancer cells. Mol Cancer Res 8: 739-750, 2010.

32. Urosevic J, Garcia-Albéniz X, Planet E, Real S, Céspedes MV, Guiu M, Fernandez E, Bellmunt A, Gawrzak S, Pavlovic M, et al: Colon cancer cells colonize the lung from established liver metastases through p38 MAPK signalling and PTHLH. Nat Cell Biol 16: 685-694, 2014.

33. Zheng L, Pu J, Jiang G, Weng M, He J, Mei H, Hou X and Tong Q: Abnormal expression of early growth response 1 in gastric cancer: Association with tumor invasion, metastasis and heparanase transcription. Pathol Int 60: 268-277, 2010.

34. Yarden Y: The EGFR family and its ligands in human cancer. signalling mechanisms and therapeutic opportunities. Eur J Cancer 37 (Supply 4): S3-S8, 2001.

35. Yonesaka K, Zejnullahu K, Okamoto I, Satoh T, Cappuzzo F, Souglakos J,Ercan D, Rogers A, Roncalli M, Takeda M, et al: Activation of ERBB2 signaling causes resistance to the EGFR-directed therapeutic antibody cetuximab. Sci Transl Med 3: 99ra86, 2011.

36. Li LY, Li EM, Wu ZY, Cao HH, Shen JH, Xu XE, Chen B, Wu JY and Xu LY: Overexpression of GRB2 is correlated with lymph node metastasis and poor prognosis in esophageal squamous cell carcinoma. Int J Clin Exp Pathol 7: 3132-3140, 2014

37. Engelman JA: Targeting PI3K signalling in cancer: Opportunities, challenges and limitations. Nat Rev Cancer 9: 550-562, 2009.

38. Urick ME, Rudd ML, Godwin AK, Sgroi D, Merino M and Bell DW: PIK3R1 (p85 $\alpha$ ) is somatically mutated at high frequency in primary endometrial cancer. Cancer Res 71: 4061-4067, 2011.

39. Cheung LW, Hennessy BT, Li J, Yu S, Myers AP, Djordjevic B, Lu Y, Stemke-Hale K, Dyer MD, Zhang F, et al: High frequency of PIK3R1 and PIK3R2 mutations in endometrial cancer elucidates a novel mechanism for regulation of PTEN protein stability. Cancer Discov 1: 170-185, 2011.

40. Yarden Y and Sliwkowski MX: Untangling the ErbB signalling network. Nat Rev Mol Cell Biol 2: 127-137, 2001. 